\title{
BMJ Open Health impacts and economic costs of residential fires (RESFIRES study): protocol for a population-based cohort study using linked administrative data
}

\author{
Lara A Harvey (D) ,, ${ }^{1,2}$ Nargess Ghassempour, ${ }^{3,4}$ Mark Whybro, ${ }^{5}$ \\ W Kathy Tannous (iD ${ }^{3,6}$
}

To cite: Harvey LA, Ghassempour N, Whybro M, et al. Health impacts and economic costs of residential fires (RESFIRES study): protocol for a population-based cohort study using linked administrative data. BMJ Open 2020;10:e037709. doi:10.1136/ bmjopen-2020-037709

- Prepublication history for this paper is available online. To view these files, please visit the journal online (http://dx.doi org/10.1136/bmjopen-2020 037709).

Received 13 February 2020 Revised 16 June 2020 Accepted 08 July 2020

Check for updates

(C) Author(s) (or their employer(s)) 2020. Re-use permitted under CC BY-NC. No commercial re-use. See rights and permissions. Published by BMJ.

For numbered affiliations see end of article.

Correspondence to

Dr Lara A Harvey;

I.harvey@neura.edu.au

\section{ABSTRACT}

Introduction Residential fires remain a significant global public health problem. It is recognised that the reported number of residential fires, fire-related injuries and deaths significantly underestimate the true number. Australian surveys show that around two-thirds of respondents who experience a residential fire are unwilling to call the fire service, and international studies highlight that many individuals who access medical treatment for fire-related injuries do not have an associated fire incident report. The objectives of this study are to quantify the incidence, health impacts, risk factors and economic costs of residential fires in New South Wales (NSW), Australia. Methods and analysis The RESFIRE cohort will include all persons living at an NSW residential address which experienced a fire over the period 2005-2014. Nine data sources will be linked to provide a comprehensive picture of individual trajectories from fire event to first responder use (fire and ambulance services), emergency department presentations, hospital admissions, burn out-patient clinic use and death. These data will be used to describe the circumstances and characteristics of residential fires, provide a profile of fire-related injuries, examine trends over time, and explore the relationship between fire circumstance, emergency and health services utilisation, and health outcomes. Regression modelling, including multilevel modelling techniques, will be used to explore factors that impact on these relationships. Costing models will be constructed.

Ethics and dissemination Ethical approval for this study has been obtained from the NSW Population and Health Service Research Ethics Committee and Western Sydney University Human Research Ethics Committee. The study reference group comprises key stakeholders including Fire and Rescue NSW, policy agencies, health service providers and burns clinicians ensuring wide dissemination of results and translation of data to inform practice and identify areas for targeted prevention. Summary reports in formats designed for policy audiences in parallel with scientific papers will be produced.

\section{INTRODUCTION}

Fire-related burns are the fourth most common cause of unintentional trauma worldwide and have been identified as a
Strengths and limitations of this study

- This large population-based retrospective cohort study will use nine administrative datasets to provide a comprehensive profile of residential fires in New South Wales over a 10-year period.

- By linking fire data to health service data, we will be able to track an individual's pathway through the fire event, first responder and healthcare use and map their health outcomes.

- Results of this study will have the potential to identify high-risk populations and to inform service planning and policy development.

- Limitations to the study design include quality of the linkage between datasets, completeness of the data and accuracy of coding.

- Individuals who only access general practitioners, medical centres or pharmacies to treat their injuries will not be included in healthcare use, potentially leading to an underestimation of the true burden of residential fires.

global priority for prevention. ${ }^{12}$ An estimated 265000 people each year die as result of fires, ${ }^{3}$ and in most industrialised countries the vast majority of fire-related deaths and injuries occur in the home. ${ }^{4}$ Fire prevention programmes that promote the use of smoke alarms are the leading strategies adopted to minimise this burden. ${ }^{25}$ Australia is among the countries that have implemented strategies and best practices in fire prevention programmes including legislation that requires functioning smoke alarms in every home. ${ }^{6}$ Despite this, residential fires remain a significant public health problem in Australia. In New South Wales (NSW), Australia's most populous state, over a 5-year period between 2013 and 2017, there were 34000 reported residential fires, resulting in 5000 injuries and 144 deaths. ${ }^{8-12}$ Estimates suggest that more than half of these may have been 
prevented if the homes had working smoke alarms and a practiced home escape plan in place. ${ }^{13}$

It is well recognised that the reported number of residential fires, fire-related injuries and deaths significantly underestimate the true number. ${ }^{8}$ Australian populationbased surveys show that around two-thirds of respondents who experience a residential fire are unwilling to call the fire service, ${ }^{14}$ and studies from the USA and New Zealand highlight that many individuals who access medical treatment for fire-related injuries do not have an associated fire incident report. ${ }^{15}$ Although most residential fire-related injury and deaths result from smoke inhalation and toxic fumes rather than burns, ${ }^{16-18}$ under the International Classification of Diseases and Related Health Problems, 10th Revision (ICD-10) classification system which is used in many administrative health data collections, smoke inhalation (ICD-10 code T59) is not included with burns (ICD-10 codes T20-T31) and hence is commonly not captured in burn-related hospital and death statistics. ${ }^{19}$ The urgent need for accurate data to inform the development and evaluation of targeted residential fire prevention strategies has been identified by the NSW Corner ${ }^{20}$ and Australian Senate. ${ }^{21}$

Similarly, the costs of residential fires extend significantly beyond damage to the property and are estimated to be about 10 times the reported cost. ${ }^{22}$ Research into the total cost of fire is defined as the losses that fire causes, directly and indirectly and the cost of provisions to prevent or mitigate the losses caused by the fire. ${ }^{23} \mathrm{In}$ Australia, the total cost of fire was estimated in 2009 to be approximately US $\$ 12$ billion per annum or $1.3 \%$ of the country's gross domestic product. ${ }^{24}$ In similar cost studies conducted overseas, it has been observed that the estimates used have wide bands of uncertainty with a need for improved methods for calculating fire structure and material performance and costs. ${ }^{25} 26$

The objectives of this study are to quantify the incidence, risk factors, health impacts and economic costs of residential fires in NSW. Using linked person-level data, this study will provide, for the first time, a comprehensive picture of first responder use (fire and ambulance services), health service utilisation (emergency department (ED), hospital in-patient and specialist burns outpatient clinics), health outcomes (length of stay (LOS), rehabilitation, readmissions and death) and economic costs. Specifically, it will address the following research questions:

1. What is the incidence, risk factors, injury profile, emergency services and health service utilisation and health outcomes for people resident at an address which has experienced a fire?

2. Do first responder and health service utilisation and health outcomes differ by type of fire, smoke alarm use, nature of injury, demographic characteristics or geographical location?

3. Has fire incidence, circumstance and fire-related injuries changed over the last decade, and if so do these trends differ between geographical location?
4. To what extent is there under-reporting of residential fires, and related injuries and deaths across the datasets?

5. What are the total economic costs, including future projected costs, associated with residential fires and residential fire-related injuries.

\section{METHODS AND ANALYSIS}

\section{Study population and cohort identification}

Just under a third of Australia's population reside in NSW, and of the 8 million residents, two-thirds live in the Sydney Metropolitan region. ${ }^{27}$ NSW has an estimated 2.8 million private dwellings, over half $(56.1 \%)$ of which are single or two person households, $32.5 \%$ are three or four person households and $11.3 \%$ contain five or more people. ${ }^{28}$ The study cohort will include all persons residing at an NSW residential address which experienced a fire in the period 1 January 2005 to 31 March 2015. In addition, any person identified as having a residential fire-related injury or death from any of the linked administrative health datasets during this period will be included in the cohort irrespective of whether there is record of residential fire.

\section{Data sources}

Nine data sources will be linked to identify the complete study cohort, and to provide information on each individual's health trajectory and costs. Datasets included and the case selection criteria for each dataset are shown in table 1 .

The Australian Computer Aided Dispatch (CAD) system records all '000' calls to emergency services requiring Fire and Rescue NSW (FRNSW) or ambulance attendance. The CAD will be used to identify all NSW residential addresses that experience a fire event for which a first responder service is called. The FRNSW Australian Incident Reporting System contains detailed information on the circumstances, management, and consequences all fires attended by FRNSW recorded in the CAD. This information includes ignition factors, area of fire, type of structure, presence or absence of functioning smoke alarm, and whether alcohol use was contributing factor, and will be used in descriptive analysis and as covariates in the models.

The NSW Ambulance datasets comprise data from the ambulance CAD system, the paper-based Patient Healthcare Record (PHCR) and electronic medical record (eMR). The eMR includes clinical and treatment information on the patients. This includes case nature or diagnosis, vital signs, management and treatment type, and result or outcome of treatment, that is, patient transportation or not and code. The PHCR contains information as per eMR and includes drug and fluid treatment, cardiac management and equipment and medication used. The CAD records have been available from July 2000, the PHCR data are available from April 2001 and the eMR data are available from 2011. 


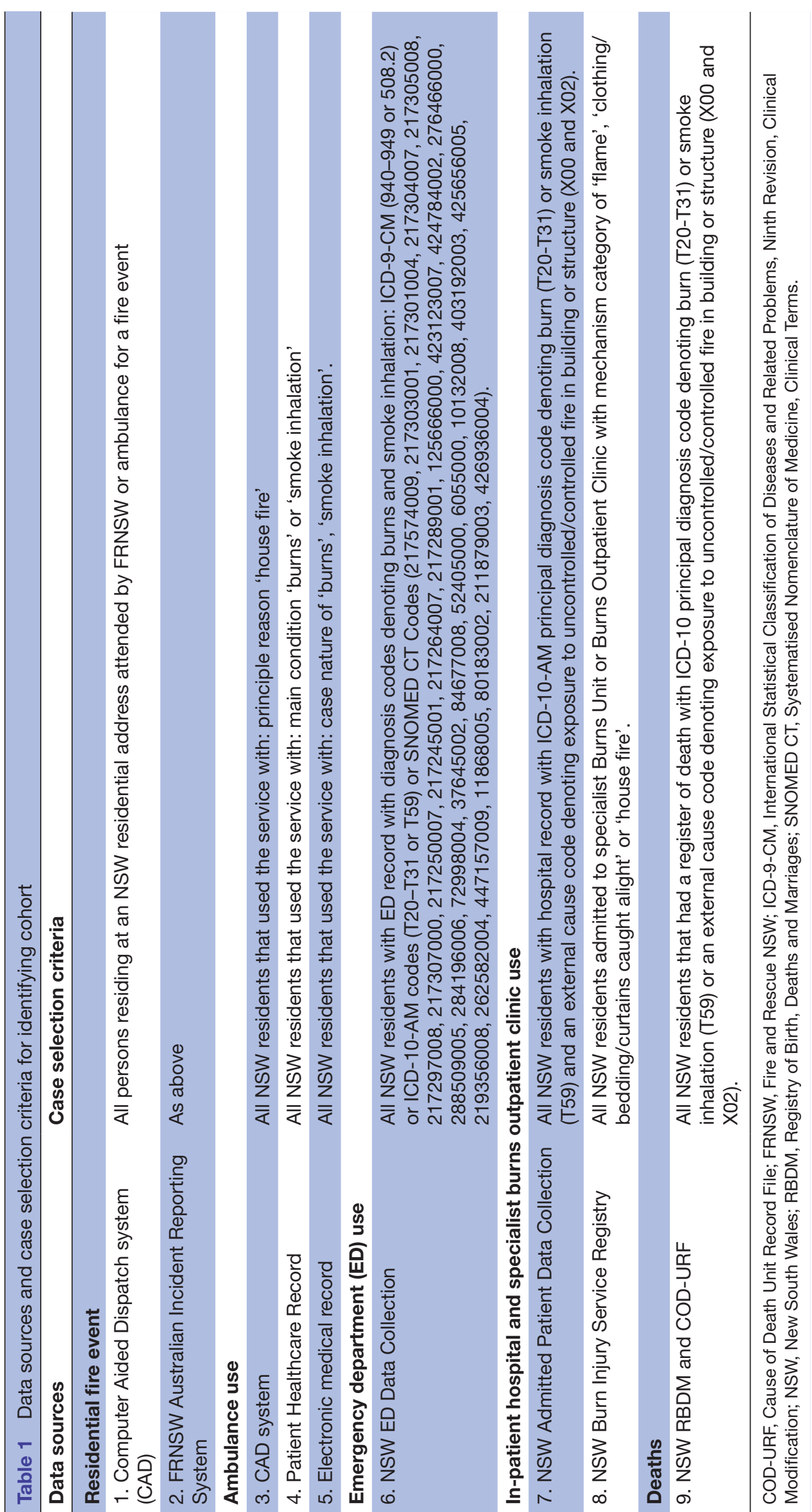


The NSW Emergency Department Data Collection (EDDC) contains information about ED presentations to NSW hospitals. The NSW EDDC covers 80 EDs, including all EDs in public hospitals in the Sydney metropolitan area and captures about $75 \%$ of all presentations to NSW EDs. The information reported includes patient demographics, mode of arrival, triage category, whether admitted to ward and presenting diagnosis. Across EDs in NSW presenting diagnosis can be recorded using the Australian version of the ICD and Related Problems, ninth revision, Clinical Modification, ${ }^{29}$ or the Australian Modification of the 10th revision, (ICD-10-AM),$^{30}$ or the Systematised Nomenclature of Medicine, Clinical Terms. ${ }^{31}$

The NSW Admitted Patient Data Collection (APDC) will be used to identify residential fire-related hospital admissions, and to provide information for risk adjustment and hospitalisation costs. These data collections contain information on demographics, diagnosis related groupings, source of referral and diagnosis and external causes coded to ICD-10-AM. Audits have shown good to excellent coding of diagnosis, procedures and external causes in Australian hospital data. ${ }^{32}{ }^{33}$ Data are collected on all episodes of care in hospital with end with the discharge, transfer or death of the patient, or when the service category for the patient changes (eg, a change from acute care to rehabilitation during a stay in the same hospital). Thus, for a single burn an individual may have multiple episodes of care recorded. To avoid overcounting of the same injury, a continuous period of stay will be constructed by combing all contiguous episodes of care for the same patient. Where episodes of care are non-contiguous, but the primary diagnoses are the same at the three-character level (ie, T20) for an individual, the hospitalisation will be considered as a readmission for the burn. Where the episodes of care are non-contiguous and the primary diagnosis and external causes indicate a different burn location and mechanism the hospitalisation will be considered as a separate burn event.

The Agency for Clinical Innovation NSW Statewide Burn Injury Service Registry contains admission records and case details for all patients admitted to the three designated Burn Units in NSW (two adult, one paediatric) and captures both hospitalisations and outpatient clinical visits. Detailed demographic, aetiological and clinical data items are collected including a free-text description of the circumstances resulting in the burn injury. Cases are classified according to mechanism of injury.

Mortality data will be obtained from the NSW Registry of Birth, Deaths and Marriages (RBDM) and the Australian Bureau of Statistics (ABS) Cause of Death Unit Record File (COD-URF). The RBDM contains records of all deaths of NSW residents, either certified by a registered medical practitioner or by the state coroner and provides date of death. Most deaths are registered within 4 weeks. The COD-URF provides additional information recorded on the death certificate including underlying COD and up to 20 contributing causes of death coded to ICD- $10 .^{34}$

\section{Data linkage}

The Centre for Health Record Linkage (CHeReL) will undertake the data linkage for this study, using probabilistic linkage techniques. Residential address from the FRNSW AIRS dataset will be used to identify cohort members across the health datasets held within the CHeReL Master Linkage Key (MLK), a system of continuously updated links between core health-related datasets in NSW. ${ }^{35}$ Personal identifiers (full name, date of birth, sex and residential address) are used to identify individuals across and within the MLK datasets. The false positive and false negative rates for linkage between MLK datasets are $0.3 \%$ and $0.5 \%$, respectively. ${ }^{36}$ The burns registry data (SIBS) which is not included in the MLK will be linked in a separate process using full name, date of birth, sex and address. The CHeReL applies a 'best practice protocol' for preserving individual privacy, which involves separating the process of linkage from the analysis of the health content. ${ }^{37}$ Data custodians provide the personal identifiers to the CHeReL, and upload the health data extracts with the unique identifier for each individual in the cohort, provided by the CHeReL, into the Secure Unified Research Environment ${ }^{38}$ for analysis by researchers.

\section{Data analysis plan}

As this study involves linkage of disparate administrative datasets, we will assess and report on the quality of the data using the ABS Data Quality Framework. ${ }^{39}$ The dimensions of quality that will be assessed are: institutional environment, relevance, timeliness, accuracy, coherence, interpretability and accessibility. In particular, it is anticipated that FRNSW AIRS data may have high degree of missing values. We will assess missingness, to determine whether data is missing completely at random, missing at random or missing not at random to ascertain whether principled methods, such as multiple imputation or inverse probability weighting are appropriate for use in our models. ${ }^{40}$ Sensitivity analysis will be conducted around our results. Linkage rates between datasets not previously linked will be reported.

\section{Research questions 1-2}

Descriptive statistics will be used to quantify the incidence, demographic and socioeconomic characteristics, service utilisation, procedures and health outcomes of persons injured as a result of a residential fire and to describe the fire circumstance and characteristics. We will use multilevel modelling to identify individual, fire characteristic and service utilisation factors associated with incidence of residential fire-related injury and health outcomes. The main health outcomes of interest are total LOS in days, LOS for acute care, LOS for rehabilitation, readmissions, 30-day mortality (fire related and all cause) and hospital costs.

Variables of interest include

Demographic and socioeconomic characteristics: age, sex, country of birth, language spoken at home, pre-existing 
health status (measured using the Charlson Comorbidity Index ${ }^{41}$ with a 1-year lookback period ${ }^{42}$ ), health insurance status and socioeconomic disadvantage (measured by the ABS Socioeconomic Indexes for Areas).$^{43}$

Geographical measures: statistical area level 2 (SA2) which is a standard geographical area used by the ABS that broadly represents a community that interacts together socially and economically. SA2s have an average population of about 10000 people (range $3000-25,000$ ) ${ }^{44}$

Injury characteristics: type of injury will be classified into-burns, smoke inhalation and musculoskeletal injuries. Other characteristics of interest include total body surface area of burn, depth of burn and procedures undertaken.

Service utilisation: number of FRNSW and ambulance attendances, ED presentations, hospital admissions and outpatient treatment.

Fire-related characteristics: nature of fire (controlled, uncontrolled, electrical, chemical), day and time of fire, type of residential structure, action taken, areas of fire origin, presence of functioning smoke alarm and/ or sprinkler system, type of smoke detector, evidence of alcohol consumption, geographical location of residence (mapped to postal code) and response agencies involved.

\section{Research question 3}

To assess changes in trend in fire incidence, incidence of fire-related injuries over time, age-specific and agestandardised rates per 100000 population with 95\% CIs will be calculated. Age-specific population estimates for NSW for 2005-2014 will be used to calculate agestandardised rates, using the Australian estimated resident population at June 2001 as the standard population. Depending on model fit Poisson or negative binomial regression will be used to examine the statistical significance of changes in trends over time.

\section{Research question 4}

Capture-recapture modelling techniques will be used to determine to what extent residential fires, and related injuries and deaths are under-reported across the datasets. Counting people captured by each system and the extent of overlap will provide the data required to run capture-recapture statistical models, ${ }^{45-47}$ overall and by demographic and other variables. These models will estimate how many people attended hospital after injuries in fires but did not call the fire or ambulance service. They also will provide an estimate of injuries sustained after a residential fire but not captured in the hospital data (eg, injuries treated in a primary care setting).

\section{Research question 5}

An injury cost model will be developed for all fires that are attended and not attended by FRNSW. Hospital admissions and number of days in hospital will be identified from the NSW APDC data. A dollar value for the cost of treatment for an individual will be calculated by multiplying the LOS (in days) for each episode of care by the daily AR-DRG cost per day provided in the National Hospital Cost Data Collection. ${ }^{48}$ The Hospital Casemix Protocol will be used to assign costs to private inpatient episodes by AR-DRG. For ED attendances, costs will be assigned using the national average ED costs based on triage category. ${ }^{49}$ The costs to individuals of the structural damage to the property will be estimated using the cost to insurance companies from the Insurance of Australia and out-of-pocket expenses. The costs of responding to the incident will be estimated based on the AIRS data on the services attended and material used. The cost of pain and suffering of individuals will be estimated using established values as per Tannous $e t a l^{50}$

A limitation of this study is that individuals who may access only general practitioners (GPs), medical centres or pharmacies to treat their injuries are not included. Primary care records will not be linked, as we are unable to detect from MBS records the reason for the GP visit, only that a GP visit occurred. However, it is worth noting that burns treated by GPs are likely to be minor in nature as there are rigorous guidelines in place for referral to a specialist outpatient burn clinic for more extensive burn injuries. Any outpatient burn clinic referral will be captured from the NSW Burn Injury Service Data, which includes outpatient visits. If people extinguish minor fires themselves and only sustain minor injuries that do not require medical treatment the health impact and economic cost is likely to be minimal.

\section{Statistical power}

The study includes whole-of population data for a 10-year period. According to FRNSW, the number of residential fires will be approximately 45,000, involving around 80000 individuals of whom over 6000 will have injuries that needed to be treated on the scene and the remainder required rescuing or evacuation. Per cent annual change in hospitalisation rates over time is our most sensitive outcome measure. Data from a previous study suggest there are approximately 70 hospital admissions per year in NSW for residential fire-related injury, with the hospitalisation rate increasing by an estimated $4.9 \%$ per year prior to the introduction of smoke alarm legislation and decreasing by $36 \%$ per year following introduction of legislation. ${ }^{7}$ Based the reported number of residential fires and hospital admissions in 2014, with an alpha of 0.05 and power of $80 \%$ the study is powered to detect a change of $2 \%$ per year, providing ample power.

\section{SIGNIFICANCE OF THIS STUDY}

In addition to contributing to the theoretical domains of fire and health economics and quantitative modelling, this research will contribute by

- Providing an in-depth profile of individuals' journey following residential fires through health services and service providers.

- Provide an in-depth profile of users of the different safety and health services. 
- Development of methodologies to collate health cost information for individuals and stakeholders impacted by fire.

- Developing an understanding of the duration of effect of fire incidents, at the individual, community service worker level as well as wider community.

The research will provide a better understanding of the impact of residential fires injuries on service usage, (FRNSW, ambulance, ED, hospital) that can be used to identify priory areas for research as well as inform and influence policy and planning in this area in NSW and beyond.

\section{ETHICS AND DISSEMINATION}

Ethical approval for this study has been provided by the NSW Population and Health Service Research Ethics Committee (HREC/16/CIPHS/36) and Western Sydney University Human Research Ethics Committee (RH12399). To ensure the translation of the study findings into policy and practice, a reference group will be established comprising policy stakeholders including representation from Fire and Rescue Services, burns unit personnel and policy bodies (Agency for Clinical Innovation). We will produce a suite of peer-reviewed manuscripts and summary reports. The reports will be in formats designed for policy audiences in parallel with presentations collaborator meetings, relevant professional conferences and public forums.

\section{Author affiliations}

${ }^{1}$ Falls, Balance and Injury Research Centre, Neuroscience Research Australia, Randwick, New South Wales, Australia

${ }^{2}$ School of Public Health and Community Medicine, University of New South Wales, Sydney, New South Wales, Australia

${ }^{3}$ School of Business, Western Sydney University, Penrith South, New South Wales, Australia

${ }^{4}$ Rozetta Insitute, Sydney, New South Wales, Australia

${ }^{5}$ Community Safety Department, Fire and Rescue New South Wales, Sydney, New South Wales, Australia

${ }^{6}$ Translational Health Research Institute, Western Sydney University, Penrith South, New South Wales, Australia

\section{Twitter Lara A Harvey @DrLaraHarvey}

Acknowledgements Support for this study is provided by the Community Safety and Research at FRNSW, Associate Professor John Harvey (Children's Hospital Westmead), Anne Darton and Siobhan Connolly (Agency for Clinical Innovation). We thank the the Centre for Health Record Linkage (CHeReL) for undertaking the probabilistic linkage and the NSW Ministry of Health for providing the health data.

Contributors WKT, LAH and MW conceived and designed the study. LAH, WKT and NG wrote and edited the manuscript. WKT, LAH, and NG provide data analytics. LAH, WKT, MW and NG reviewed and approved the final version of the protocol.

Funding This work was supported by Fire and Rescue NSW grant [P00024777] towards the cost of data linkage, and Secured Unified Research Environment upload, site management and data access for WKT, LAH and NG.

\section{Competing interests None declared.}

Patient and public involvement Patients and/or the public were not involved in the design, or conduct, or reporting, or dissemination plans of this research.

Patient consent for publication Not required.

Provenance and peer review Not commissioned; externally peer reviewed.
Open access This is an open access article distributed in accordance with the Creative Commons Attribution Non Commercial (CC BY-NC 4.0) license, which permits others to distribute, remix, adapt, build upon this work non-commercially, and license their derivative works on different terms, provided the original work is properly cited, appropriate credit is given, any changes made indicated, and the use is non-commercial. See: http://creativecommons.org/licenses/by-nc/4.0/.

ORCID iDs

Lara A Harvey http://orcid.org/0000-0001-6260-8001

W Kathy Tannous http://orcid.org/0000-0002-3153-5652

\section{REFERENCES}

1 World Health Organization. Injuries and violence: the facts 2014. Geneva: WHO, 2014.

2 World Health Organization. Burn Prevention: Success stories, lessons learned. Geneva: WHO, 2011.

3 World Health Organization. Burns. Fact sheets, 2018. Available: https://www.who.int/violence_injury_prevention/other_injury/burns/ en/ [Accessed Feb 2020].

4 TriData Division. Global concepts in residential fire safety: part 3best practices from Canada, Puerto Rico, Mexico and Dominican Republic. Arlington VA System Planning Corporation; 2009.

5 Ahrens M. Home structure fires. Quincy, MA: National Fire Protection Association, 2013.

6 TriData Division. Global concepts in residential fire safety: part 2best practices from Australia, New Zealand and Japan. Arlington VA System Planning Corporation; 2008.

7 Harvey LA, Poulos RG, Sherker S. The impact of recent changes in smoke alarm legislation on residential fire injuries and smoke alarm ownership in New South Wales, Australia. J Burn Care Res 2013;34:e168-75.

8 Fire and Rescue New South Wales. Submission to Commonwealth Senate Inquiry into Smoke Alarms. Sydney: FRNSW, 2015.

9 Productivity Commission. Report on Government Services 2016. Canberra: Australian Government, 2016.

10 Productivity Commission. Report on government services 2017. Canberra: Australian Government, 2017.

11 Productivity Commission. Report on Government Services 2018. Canberra: Australian Government, 2018.

12 Productivity Commission. Report on Government Services 2019. Canberra: Australian Government, 2019.

13 Fire and Rescue New South Wales. Annual report 2014/15. Sydney: FRNSW, 2015.

14 Tannous WK, Agho K. Socio-Demographic predictors of residential fire and unwillingness to call the fire service in New South Wales. Prev Med Rep 2017;7:50-7.

15 Chubb M. Wake up and smell the smoke. Fire Engineering, 2003.

16 Fire Brigades NSW. Fire Fatalities report - study of fatal fires in NSW from 2004 to 2008. Sydney: NSW Fire Brigade, 2009.

17 Edelman LS. Social and economic factors associated with the risk of burn injury. Burns 2007;33:958-65.

18 Atiyeh BS, Costagliola M, Hayek SN. Burn prevention mechanisms and outcomes: pitfalls, failures and successes. Burns 2009;35:181-93.

19 Harvey LA, Poulos RG, Finch CF. Making burns count: the impact of varying case selection criteria on the identification of ICD-10 coded hospitalised burns. Burns 2013;39:1367-73.

20 Dillon H. Finding of inquest into the death of Connie Zhang injuiry into fire at Unit 53, 4 West Terrace, Bankstown. Coronors Court New South Wales, 2015.

21 Senate Legal and Constitutional Affairs Committee. Use of smoke alarms to prevent smoke and fire related deaths. Canberra: NSW Government, 2016.

22 Hall JR. The total cost of fire in the United States, 2013. Quincy, MA: National Fire Protection Association, 2013.

23 Ashe B, McAneney J, Pitman A. Is the allocation of resources towards mitigation and response to fire in Australia optimal? J Risk Res 2011;14:381-93.

24 Ashe B, McAneney KJ, Pitman AJ. Total cost of fire in Australia. J Risk Res 2009;12:121-36.

25 Hall JR. The total cost of fire in the United States, 2011. Quincy, MA: National Fire Protection Association, 2011.

26 Australian Building Codes Board. Building Code of Australia 2006. Canberra: ABC Board, 2006.

27 Australian Bureau of Statistics. Estimated Residential Population, Australian Demographic Statistics. Canberra: ABS, 2019.

28 Australian Bureau of Statistics. Census of Population and Housing 2016. Canberra: ABS, 2016. 
29 National Coding Centre. Australian Version of the International Classification of Diseases, Ninth Revision, Clinical Modificiation (ICD9-CM). Second edition. Sydney: University of Sydney, 1996.

30 National Centre for Classification in Health. The International statistical classification of diseases and related health problems, tenth revision, Australian modification (ICD-10-AM. Canberra: Commonwealth of Australia, 2010.

31 The International Health Terminology Standards Development Organisation. Systemized Nomenclature of Medical Clinical Terms (SNOMED-CT) 2002.

32 Henderson T, Shepheard J, Sundararajan V. Quality of diagnosis and procedure coding in ICD-10 administrative data. Med Care 2006;44:1011-9.

33 McKenzie K, Enraght-Moony EL, Waller G, et al. Causes of injuries resulting in hospitalisation in Australia: assessing coder agreement on external causes. Inj Prev 2009;15:188-96.

34 World Health Organization. The International Statistical Classification of Diseases and Related Health Problems, Tenth Revision (ICD-10). 2nd edn. Geneva: WHO, 2004.

35 Centre for health record linkage. Master linkage key. Available: https://www.cherel.org.au/master-linkage-key [Accessed Feb 2020].

36 Centre for Health Record Linkage. Master linkage key: quality assurance report. Sydney: CHeReL, 2012.

37 Kelman CW, Bass AJ, Holman CDJ. Research use of linked health data--a best practice protocol. Aust N Z J Public Health 2002;26:251-5.

38 Sax Institute. Sure, 2019. Available: https://www.saxinstitute.org.au/ our-work/sure/

39 Australan Bureau of Statistics. 1520.0 - ABS data quality framework. Canberra: ABS, 2009. https://www.abs.gov.au/ausstats/abs@.nsf/ Latestproducts/1520.0
40 Perkins NJ, Cole SR, Harel O, et al. Principled approaches to missing data in epidemiologic studies. Am J Epidemiol 2018;187:568-75.

41 Quan $\mathrm{H}$, Sundararajan V, Halfon P, et al. Coding algorithms for defining comorbidities in ICD-9-CM and ICD-10 administrative data. Med Care 2005;43:1130-9.

42 Preen DB, Holman C D'Arcy J, Spilsbury K, et al. Length of comorbidity lookback period affected regression model performance of administrative health data. J Clin Epidemiol 2006;59:940-6.

43 Australian Bureau of Statistics. Socio-economic Indexes for Area (SEIFA) - technical paper 2006. Canberra: ABS, 2008.

44 Australian Burea of Statistics. 1270.0.55.001 Australian Statitcical geography standard (ASGS): volume 1 main structure and greater capital City statistical areas. Canberra: ABS, 2016.

45 Chao A, Tsay PK, Lin SH, et al. The applications of capture-recapture models to epidemiological data. Stat Med 2001;20:3123-57.

46 Hassel M, Asbjørnslett BE, Hole LP. Underreporting of maritime accidents to vessel accident databases. Accid Anal Prev 2011;43:2053-63.

47 Miller TR, Gibson R, Zaloshnja E, et al. Underreporting of driver alcohol involvement in United States police and hospital records: capture-recapture estimates. Ann Adv Automot Med 2012;56:87-96.

48 Independent Hospital Pricing Authority. National Hospitals Cost data Collection, Round 21. Sydney: IPHA, 2017.

49 Australian Institute of Health and Welfare. Admitted patient care 2015-16: Australian hospital statistics. Canberra: AlHW, 2017.

50 Tannous WK, Whybro M, Lewis C, et al. Home fire safety checks in New South Wales: an economic evaluation of the pilot program. $J$ Risk Res 2018;21:1052-67. 